\title{
Velocity-locked solitary waves in quadratic media
}

Fabio Baronio, Matteo Conforti, Costantino De Angelis

CNISM, Università di Brescia, Via Branze 38, 25123 Brescia, Italy

\author{
Antonio Degasperis \\ Dipartimento di Fisica and INFN, Università "La Sapienza", P.le A. Moro 2, 00185 Roma, Italy \\ Marco Andreana, Vincent Couderc, Alain Barthélémy \\ XLIM, CNRS and Université de Limoges, Av. Albert Thomas 123, 87060, Limoges, France
}

(Dated: September 11, 2018)

\begin{abstract}
We demonstrate experimentally the existence of three-wave resonant interaction solitary triplets in quadratic media. Stable velocity-locked bright-dark-bright spatial solitary triplets, determined by the balance between the energy exchange rates and the velocity mismatch between the interacting waves, are excited in a KTP crystal.

PACS numbers: 42.65.Tg, 05.45.Yv, 42.65.Sf
\end{abstract}

In recent years solitary waves in quadratic materials have been the subject of an intense renewal of interest from both theoretical and experimental viewpoints. Two types of solitary waves that were both predicted in the early 1970's are being studied. On one hand, one finds solitary waves that result from a balance between nonlinearity and diffraction (or dispersion) [1]. This type of solitary wave has been intensively investigated experimentally over the past few years [2]. On the other hand, quadratic media were shown to support solitary waves that result from energy exchanges between diffractionless (or dispersionless) waves of different velocities [3 $\left[\begin{array}{l}5 \\ \text {. }\end{array}\right.$. The structure of these solitary waves is determined by the balance between the energy exchange rates and the velocity mismatch between the interacting waves [6, 7]. This type of solitary wave is ubiquitous in nonlinear wave systems [5] and has been reported in such diverse fields as plasma physics, hydrodynamics, acoustics, and nonlinear optics, in particular in the context of self-induced transparency [4, 8]. This type of wave has also been investigated experimentally in stimulated Raman scattering in gases [9] and recently in $\mathrm{H}_{2}$-filled photonic crystal fibers [10], in stimulated Brillouin fiber-ring lasers [11], but no experiments have been reported to date on solitary waves of quadratic optical materials.

In this Letter we report the experimental observation of diffractionless velocity-locked solitary triplets in a quadratic crystal. We consider the optical spatial noncollinear scheme with type II second harmonic generation (SHG) in a KTP crystal. A spatial narrow diffractionless extraordinary beam (the signal) and an ordinary quasi-plane wave (the pump), both at the fundamental frequency $(\mathrm{FF}) \omega$, mix via $\chi^{(2)}$ to generate a second harmonic ( $\mathrm{SH}$ ) beam at frequency $2 \omega$ (the idler). Depending on the input intensities, three different regimes exist. Linear regime: the FF signal and pump beams do not interact. Frequency conversion: the FF signal and pump beams interact and generate a SH idler whose spatial characteristics are associated with the interaction distance in the crystal; signal and pump are depleted.
Solitary regime: the FF signal and pump beams interact, generate a spatial narrow $\mathrm{SH}$ idler, a spatial dip appears in the pump, whereas the intensity and propagation direction of the signal beam are modified. Indeed, the interaction generates a stable bright-dark-bright triplet moving with a locked spatial nonlinear velocity 7].

In the experiments (see Fig. 1), a Q-switched, modelocked Nd:YAG laser delivers $40 \mathrm{ps}$ pulses at $\lambda=1064 \mathrm{~nm}$. We introduce a Glan polarizer to obtain, after passage of the light through $P_{1}$, two independent beams with perpendicular linear polarization states. A half-wave plate placed before the prism serves to adjust the intensity of the two beams. By means of highly reflecting mirrors, beam splitters and lenses the beams are focused and spatially superimposed in the plane of their beam waist with a circular shape of $200 \mu \mathrm{m}$ and $2.2 \mathrm{~mm}$, full width at half maximum in intensity, for the signal and pump waves respectively. A $L=3 \mathrm{~cm}$ long type II KTP crystal cut for second harmonic generation is positioned such that its input face corresponds to the plane of superposition of the two input beams. The crystal is oriented for perfect

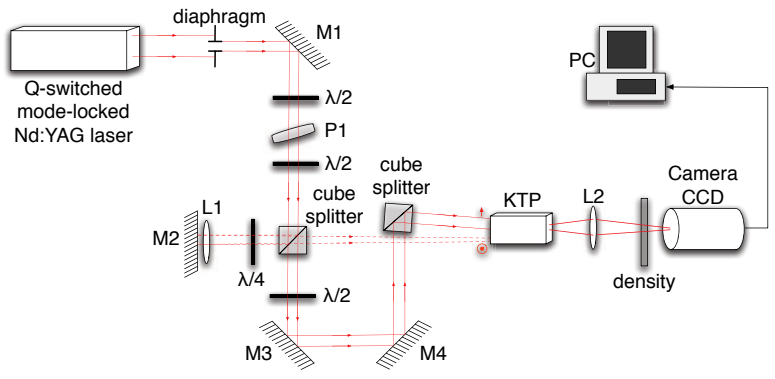

FIG. 1: Experimental set-up. M1, M2, M3, M4: mirrors. P1: polarizer. L1, L2: lenses.

phase matching. The directions of the linear polarization state of the two beams are adjusted to coincide with the extraordinary and the ordinary axes, respectively, of 
the KTP crystal. The wave vectors of the input beams are tilted at angles of $\theta_{s}=2.1^{\circ}$ and $\theta_{p}=-2.1^{\circ}$ (in the crystal) with respect to the direction of perfect collinear phase matching for the extraordinary and the ordinary components, respectively (see Fig. 2). These parameters correspond inside the crystal to a tilt between the input beams greater than the natural walk-off angle but introduced along the ordinary noncritical plane. The idler second harmonic direction lies in between the input beams directions $\left(\theta_{i} \cong 0.4^{\circ}\right)$. With these values of parameters, spatial diffraction and temporal dispersion were negligible. The spatial waves' patterns at the output of the crystal are imaged with magnification onto a CCD camera and analyzed. We use alternately different filters and polarizers to select either the IR or the green output.

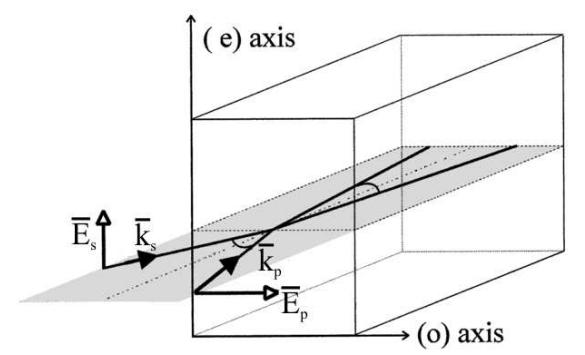

FIG. 2: Schematic representation the optical non collinear configuration in the KTP crystal.

Theoretically, the equations describing the spatial quadratic resonant interaction of three waves in the quadratic nonlinear medium read as:

$$
\begin{aligned}
& \left(\frac{\partial}{\partial z}-\rho_{s} \frac{\partial}{\partial x}\right) E_{s}+\frac{1}{2 i k_{s}}\left(\frac{\partial^{2}}{\partial x^{2}}+\frac{\partial^{2}}{\partial y^{2}}\right) E_{s}=i \chi_{s} E_{p}^{*} E_{i} \\
& \left(\frac{\partial}{\partial z}-\rho_{p} \frac{\partial}{\partial x}\right) E_{p}+\frac{1}{2 i k_{p}}\left(\frac{\partial^{2}}{\partial x^{2}}+\frac{\partial^{2}}{\partial y^{2}}\right) E_{p}=i \chi_{p} E_{s}^{*} E_{i} \\
& \left(\frac{\partial}{\partial z}-\rho_{i} \frac{\partial}{\partial x}\right) E_{i}+\frac{1}{2 i k_{i}}\left(\frac{\partial^{2}}{\partial x^{2}}+\frac{\partial^{2}}{\partial y^{2}}\right) E_{i}=i \chi_{i} E_{s} E_{p}
\end{aligned}
$$

$E_{j}(x, y, z)$ are the slowly varying electric field envelopes of the waves at frequencies $\omega_{j}$ (wavelength $\lambda_{j}$ ), $k_{j}=$ $\omega_{j} n_{j} / c$ are the wavenumbers, $n_{j}$ the refractive indexes, $\chi_{j}=2 d \omega_{j} / c n_{j}$ the nonlinear coupling coefficients $(d$ is the quadratic nonlinear susceptibility and $c$ is the speed of light), $\rho_{j}$ the walk off angles and $j=s, p, i$ (s:signal, p:pump, i:idler). $z$ is the spatial longitudinal propagation coordinate, $x$ and $y$ are the spatial transverse coordinates. In the configuration we considered $\rho_{p}<\rho_{i}<\rho_{s}$, spatial diffraction is negligible; therefore, Eqs. (11) reduce to the integrable three-wave model reported in Ref. [7], and in the ordinary $x-z$ plane $(y=0$ plane) brightdark-bright triplets that travel with a common nonlinear locked velocity can be excited. Typical envelope profiles of the bright-dark-bright solitary waves of Eqs. (11) versus $x$ for fixed $z$ and $y$ are shown in Fig. 3 .

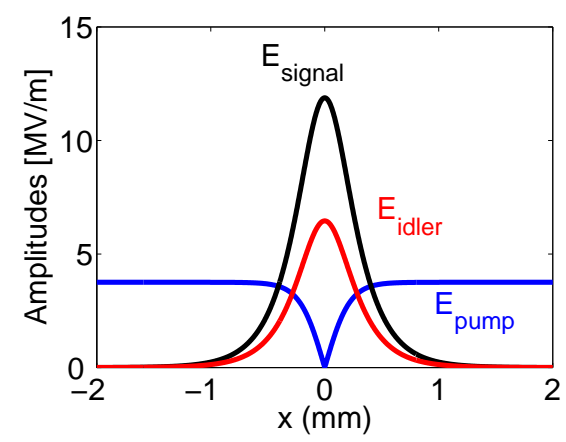

FIG. 3: Envelopes $E_{s}, E_{p}$ and $E_{i}$ of the solitary triplet.

As the intensities of the input signal and pump are varied in a suitable range, we observed three different regimes in the ordinary $\mathrm{KTP} x-z(y=0)$ plane: linear, frequency conversion, and solitary regimes.

In the low-intensity linear regime $\left(I_{s}=0.1 \mathrm{MW} / \mathrm{cm}^{2}\right.$, $I_{p}=0.01 M W / \mathrm{cm}^{2}$ ), the signal and the pump do not interact and propagate without diffraction in the KTP crystal following their own characteristic spatial directions (Fig. 4). Left column of Fig. 4 shows the numerical spatial evolution of the extraordinary polarized signal and the ordinary polarized pump in the ordinary $x-z(y=0)$ plane; central and right columns report respectively the numerical and experimental spatial output profiles of the beams in the $x-y(z=L)$ plane. The numerical and experimental results are reported considering a spatial frame moving with the pump walk off angle. No SH extraordinary polarized idler is generated.
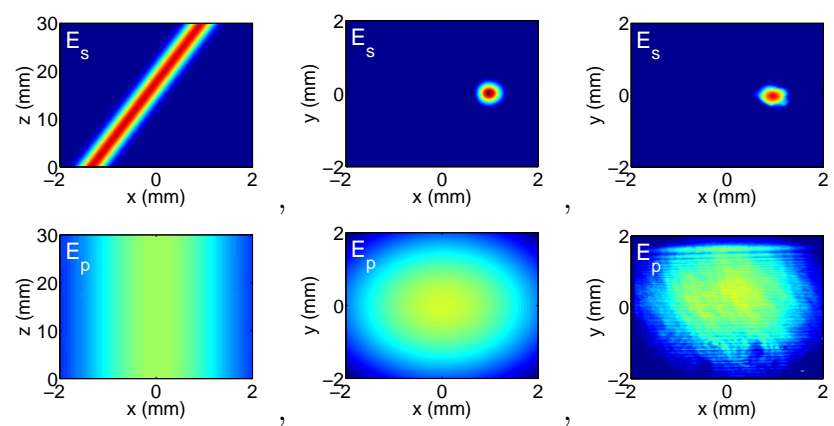

FIG. 4: Linear regime. Left column, numerical dynamics of the beams $E_{s}, E_{p}$ in the $x-z(y=0)$ plane. Central column, numerical, and right column, experimental results at the exit face of the KTP crystal presenting the spatial $x-y$ output profiles of the beams. At the input $I_{s}=0.1 \mathrm{MW} / \mathrm{cm}^{2}$, $I_{p}=0.01 M W / \mathrm{cm}^{2}$.

At moderate input intensities $\left(I_{s}=10 \mathrm{MW} / \mathrm{cm}^{2}, I_{p}=\right.$ $\left.0.03 \mathrm{MW} / \mathrm{cm}^{2}\right)$, the signal interacts with the pump and an idler beam at the second harmonic is generated (Fig. 
5). This regime corresponds to the well known optical non-collinear second harmonic frequency conversion. As shown in the left column of Fig. 5, the signal beam and the pump beam propagate with their own spatial velocities (walk off angles); as long as the signal beam overtakes the pump beam, a SH idler beam is generated which propagates with its own characteristic spatial linear velocity; the spatial width of the SH idler is associated with the FF beams' interaction distance in the crystal. Signal and pump beams are deeply depleted. Central and right columns of Fig. 5 report respectively the numerical and the experimental spatial output profiles of the beams in the $x-y(z=L)$ plane. Indeed, central and right columns of Fig. 5 report the existence of both the linear and the frequency conversion regimes. In the planes parallel to the $x-z(y=0)$ plane, low-intensity tails of the signal, along the $y$ coordinate, lead to linear beamdynamics along the $x$ dimension (as in the $y=1$ plane), while moderate-intensity levels of the signal, along the $y$ coordinate, lead to frequency conversion regime along the $x$ dimension (as in the $y=0$ plane).
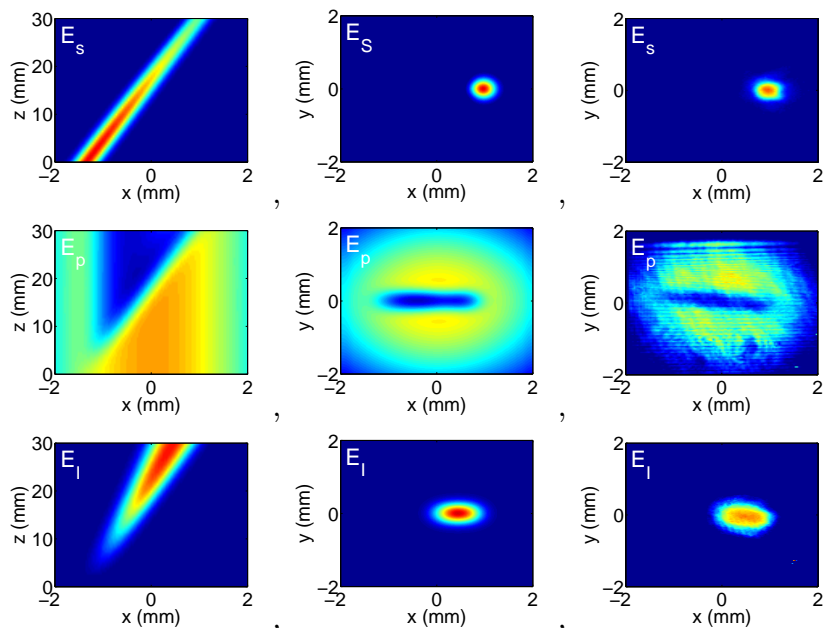

FIG. 5: Frequency conversion. Left column, numerical dynamics of the beams $E_{s}, E_{p}, E_{i}$ in the $x-z(y=0)$ plane. Central column, numerical, and right column, experimental results at the exit face of the KTP crystal presenting the spatial $x-y$ output profiles of the beams. At the input $I_{s}=10 \mathrm{MW} / \mathrm{cm}^{2}, I_{p}=0.03 \mathrm{MW} / \mathrm{cm}^{2}$.

At high input intensities $\left(I_{s}=50 \mathrm{MW} / \mathrm{cm}^{2}, I_{p}=\right.$ $0.1 \mathrm{MW} / \mathrm{cm}^{2}$ ), the scenario changes dramatically (Fig. 6). As shown in the left column of Fig. 6 the interaction of the signal and pump beams leads to the generation of a narrow SH beam. Additionally, a narrow dip appears in the quasi-plane wave pump; the intensity, width and spatial direction of the signal are slightly modified [12]. The signal-pump interaction generates a stable brightdark-bright solitary triplet moving with a locked spatial nonlinear velocity (nonlinear walk off angle) that lies in between the characteristic spatial linear velocities of the signal and the idler [7, 13]. The solitary wave results from energy exchanges between diffractionless waves of different spatial velocities.

Central and right columns of Fig. 6) report respectively the numerical and the experimental spatial output profiles of the beams in the $x-y(z=L)$ plane. Again, central and right columns of Fig. 6 report the existence of the linear regime, the frequency conversion regime and the solitary regime. In the planes parallel to the $x-z$ $(y=0)$ plane, low-intensity tails of the signal, along the $y$ coordinate, lead to linear beam-dynamics along the $x$ dimension (as in the $y=1$ plane); moderate-intensity levels of the signal, along the $y$ coordinate, lead to frequency conversion regime along the $x$ dimension (as in the $y=0.2$ plane); while high-intensity peaks of the signal, along the $y$ coordinate, lead to solitary regime along the $x$ dimension (as in the $y=0$ plane). An effective
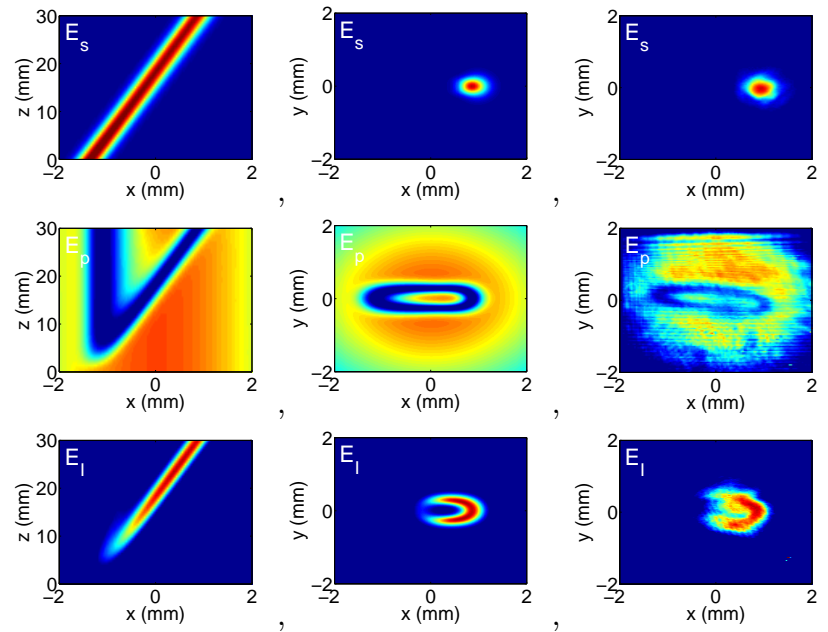

FIG. 6: Solitary regime. Left column, numerical dynamics of the beams $E_{s}, E_{p}, E_{i}$ in the $x-z(y=0)$ plane. Central column, numerical, and right column, experimental results at the exit face of the KTP crystal presenting the spatial $x-y$ output profiles of the beams. At the input $I_{s}=50 \mathrm{MW} / \mathrm{cm}^{2}$, $I_{p}=0.1 \mathrm{MW} / \mathrm{cm}^{2}$.

way to observe the spatial shift of the directions of the waves between moderate and high-intensity levels is to look at the experimental and numerical spatial output profile of the generated $E_{i}$ component in Fig. 6. In fact, we note an horseshoe-shape of the $E_{i}$ component. The lateral sides of the horseshoe represent $E_{i}$ waves generated by moderate-intensity levels of $E_{s}$; the generated $E_{i}$ waves move with the linear walk off angle $\rho_{i}$. The central portions of the horse-shoe represent $E_{i}$ waves generated by high-intensity levels of $E_{s}$; the $E_{i}$ waves move with nonlinear walk off angles within the range $\left[\rho_{i}, \rho_{s}\right]$. The nearest to the centre the highest the nonlinear walk off.

By increasing or decreasing the signal and/or pump intensities we can observe that stable solitary triplet with different nonlinear spatial velocity, width and energy distributions may be excited.

Note that the case reported in this paper is completely 
different from the quadratic walking solitons already discussed in the literature in the presence of non negligible diffraction [14, 15]. In the same way, our triplets are completely different from Manakov-type vector solitons [16, 17], where a cubic nonlinearity balances diffraction.

In summary, we have shown the existence of optical solitary waves sustained by phase-matched nondegenerate three-wave parametric interaction in a quadratic KTP medium. These solitary waves, predicted in the 1970's, are stable velocity-locked bright- dark-bright spatial triplets, determined by the balance between the energy exchange rates and the velocity mismatch between the interacting waves. It is interesting to notice that the three-wave solitary triplet concept may be applied to describe the interaction between either three beams in the spatial domain (diffractionless solitary waves) or three optical pulses in the time domain (dispersionless solitary waves).

The present research in Brescia is supported by the MIUR project PRIN 2007-CT355C.
[1] Y.N. Karamzin and A.P. Sukhorukov, JEPT Lett. 20, 339 (1974); Sov. Phys. JEPT 41, 414 (1976).

[2] A. Buryak, P. Di Trapani, D. Skryabin, and S. Trillo, Phys. Rep. 370, 63 (2002).

[3] J. A. Armstrong, S. S. Jha, and N. S. Shiren, IEEE J. Quantum Electron. QE-6, 123 (1970).

[4] K. Nozaki and T. Taniuti, J. Phys. Soc. Jpn. 34, 796 (1973).

[5] D. J. Kaup, A. Reiman, and A. Bers, Rev. Mod. Phys. 51, 275 (1979).

[6] S. Trillo, Opt. Lett. 21, 1111 (1996).

[7] A. Degasperis, M. Conforti, F. Baronio, and S. Wabnitz, Phys. Rev. Lett. 97, 093901 (2006).

[8] S. McCall and E. Hahn, Phys. Rev. Lett. 18, 908 (1967).

[9] K. Druhl, R. Wenzel, and J. Carlsten, Phys. Rev. Lett. 51, 1171 (1983)

[10] A. Abdolvand, A. Nazarkin, A. Chugreev, C. Kaminski, and P. Russel, Phys. Rev. Lett. 103, 183902 (2009).

[11] E. Picholle, C Montes, C. Leycuras, O. Legrand, and J. Botineau, Phys. Rev. Lett. 66, 1454 (1991).

[12] M. Conforti, F. Baronio, A. Degasperis, and S. Wabnitz, Opt. Express 15, 12246 (2007).

[13] M. Conforti, F. Baronio, A. Degasperis, and S. Wabnitz, Phys. Rev. E 74, 065602(R) (2006).

[14] L. Torner, W. Torruellas, G. Stegeman, and C. Menyuk, Opt. Lett. 20, 1952 (1995).

[15] W. Torruellas, G. Assanto, B. Lawrence, R. Fuerst, and G. Stegeman, Appl. Phys. Lett. 68, 1449 (1996).

[16] M. Shalaby and A. Barthelemy, IEEE J. Quantum Electron. 28, 2736 (1992).

[17] J. U. Kang, G. I. Stegeman, J. S. Aitchison, and N. Akhmediev, Phys. Rev. Lett. 76, 3699 (1996). 